\title{
A numerical design approach for single amplifier, Active-RC Butterworth filter of order 5
}

\section{Gaunholt, Hans}

Published in:

Proceedings of the IEEE International Symposium on Circuits and Systems

Link to article, DOI:

10.1109/ISCAS.2007.378352

Publication date:

2007

Document Version

Publisher's PDF, also known as Version of record

Link back to DTU Orbit

Citation (APA):

Gaunholt, H. (2007). A numerical design approach for single amplifier, Active-RC Butterworth filter of order 5 . In Proceedings of the IEEE International Symposium on Circuits and Systems (pp. 1927-1930)

https://doi.org/10.1109/ISCAS.2007.378352

\section{General rights}

Copyright and moral rights for the publications made accessible in the public portal are retained by the authors and/or other copyright owners and it is a condition of accessing publications that users recognise and abide by the legal requirements associated with these rights.

- Users may download and print one copy of any publication from the public portal for the purpose of private study or research.

- You may not further distribute the material or use it for any profit-making activity or commercial gain

- You may freely distribute the URL identifying the publication in the public portal

If you believe that this document breaches copyright please contact us providing details, and we will remove access to the work immediately and investigate your claim 


\section{A numerical design approach for single amplifier, Active-RC Butterworth filter of order 5}

\author{
H.Gaunholt, \\ Electronics and Signal Processing \\ Technical University of Denmark \\ 2800 Lyngby, Denmark
}

\begin{abstract}
A design method is presented for the design of all pole lowpass active-RC filters applying operational amplifiers. The operational amplifier model used is the integrator model: $\omega_{t} / s$ where $\omega_{t}$ is the unity gain frequency. The design method is used for the design of a fifth order Butterworth filter applying just one operational amplifier coupled as a unity gain amplifier. It is shown that the influence from the real operational amplifier may be reduced by trimming just one resistor in the circuit. The unity gain amplifiers have the advantage of providing low power consumption, yielding a large dynamic range, sometimes simplifying the amplifier design and being usable over a larger frequency range than conventional constant gain amplifiers. The Schoeffler sensitivity index is used as a basis for a sensitivity comparison with other similar filters reported in the literature.
\end{abstract}

\section{INTRODTION}

Single-amplifier biquads are most often used for building higher order active filters by using the cascade technique. In order to reduce the amount and complexity of the active circuitry required it will be an advantage to design higher order active filters by using only one operational amplifier. In this way it is possible to have low power consumption, low noise and what is shown in this article to have reasonable low sensitivities. Unfortunately the analytical solution of the design equations for filters of higher order than two becomes very difficult if not unsolvable. In this paper we will present a design technique for the solution of the design equations without the need of computing the transfer function coefficients analytically. An analytical computation of the transfer function coefficient would have been necessary following the method described in [2] and [4]. The proposed method is general and may be used to design active filters applying real operational amplifiers. Real operational amplifiers may be described by the input and output impedances as well as the unity gain frequency. As the unity gain frequency is the key parameter of the integrator model of the operational amplifier we will in this paper neglect the influence of the input and output impedances.
Similar attempts to design higher order active RC-filters with one operational amplifier are reported in [1] and [4]. In the approach described in [1] an increased amplifier gain has been used to reduce the parameter spreading but with an increased sensitivity as a result. Kramer in [4] has reported interesting results by numerically solving the design equations for third order filters with the constraints of minimizing the spread of component values. In both cases ideal operational amplifiers are used.

\section{THE DESIGN METHOD}

Consider a transfer function $\mathrm{H}(\mathrm{s})$ of a linear filter:

$$
H(s)=\frac{B(s)}{A(s)}
$$

$B(s)$ is the numerator polynomial and $A(s)$ is the denominator polynomial. The transfer function $H(s)$ which is called the target rational function is factored in the following form

$$
H(s)=H_{0} \frac{\prod_{k=1}^{m}\left(s-s_{z k}\right)}{\prod_{j=1}^{n}\left(s-s_{p j}\right)}
$$

with $m$ zeros $s_{z k}$ and $n$ poles $s_{p j}$. The real constant $H_{0}$ is a scale factor.

If we assume that all the zeros and poles of $H(s)$ are simple then we can formulate the following scalar error function $\varepsilon(\mathbf{x})$

$$
\varepsilon(\mathbf{x})=\sum_{j=1}^{n} \frac{1}{\left|H\left(\mathbf{x}, s_{p j}\right)\right|^{2}}+\sum_{k=1}^{m}\left|H\left(\mathbf{x}, \mathrm{s}_{\mathrm{zk}}\right)\right|^{2}+\sum_{i=1}^{\ell}\left\{\left|H\left(\mathbf{x}, j \omega_{i}\right)\right|-\left|H\left(j \omega_{i}\right)\right|\right\}^{2}
$$

where $H(s)$ is the target rational function and $H(\mathbf{x}, s)$ is the realized rational function. $\mathbf{x}$ is the vector of variable parameters e.g. resistors and capacitors of the circuit or unity gain frequencies of the operational amplifiers. The last term which utilizes some additional points of the magnitude of $H(s)$ at real frequencies $\omega_{i}$ is used if a specified output level is wanted. One point will normally be sufficient but more points may be incorporated if $H(s)$ contains multiple poles and zeros. The evaluation of $\varepsilon(\mathbf{x})$ could be done by any general purpose circuit simulation program. This program should have the possibility of evaluating the network function $H(\mathbf{x}, s)$ in the whole s-plane. 
If the function $\varepsilon(\mathbf{x})$ from equation (3) has been reduced to zero a match of the target function $H(s)$ and the realized function $H(\mathbf{x}, S)$ has been obtained i.e. they have the same poles and zeros. The evaluation of $\varepsilon(\mathbf{x})$ requires only knowledge of the explicit form of $H(s)$ in terms of poles and zeros as well as the structure and initial component values of the circuit to be designed. We don't need the network function with the coefficients in analytical form. This analytical form is required when setting up the design equations for active filters by following the procedure described in [2], [3] and [4].

Any optimization strategy may be useful in reducing the function $\varepsilon(\mathbf{x})$ to zero, but it is recommended to use the damped least square algorithms due to Levenberg [8] and Marquardt [9]. This algorithm in a modified form has been described by Fletcher in [7]. Another very useful modification has been described by the author in [5]. This modification is a way of changing the damping constant from iteration to iteration allowing the algorithm to take diverging iterations when the normal equations of the linear least square problem tend to be ill-conditioned. This modification has meant a considerable improvement of the overall convergence rate especially for difficult optimization problems.

The whole analysis and optimization process is coded in FORTRAN. In our approach the network equations are set up from a net list and solved for each of the poles and zeros appearing in the problem at hand. Net list is used in the same way by the SPICE program. This is a very flexible approach making it possible to change the structure and filter response without doing any additional coding. A typical run with 50 iterations takes less than $1 \mathrm{sec}$ on a personal computer.

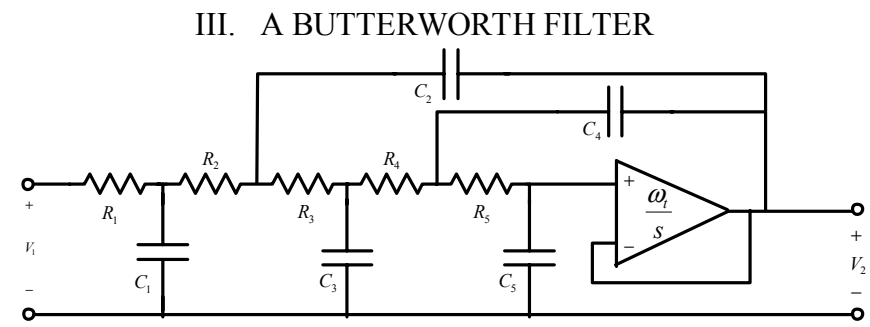

Figure 1: Fifth order unity gain all-pole filter

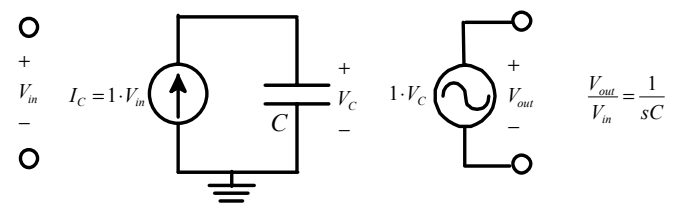

Figure 2: Operational Amplifier Model

To illustrate the utility of the proposed design method we have used it to design a $5^{\text {th }}$ order low-pass filter of the Butterworth type. The structure is an extended Sallen-Key filter applying only one operational amplifier and is shown in Fig.1. The operational amplifier is coupled as a unity gain amplifier since unity gain amplifiers have the advantage of providing low power consumption, yielding a large dynamic range, simplifying the amplifier design and being usable over a larger frequency range than more conventional constant gain amplifiers. The conventional way of designing such a filter would be the cascade approach where the Butterworth transfer function is realized as a cascade connection of one first order section and two second order sections. As the example will show the extended Sallen-Key structure offers some distinct advantage as compared with the cascade approach.

\begin{tabular}{|c|c|c|c|}
\hline & $\begin{array}{c}\text { Ideal } \\
\text { Op.Amp. }\end{array}$ & $\begin{array}{c}\text { Op.Amp. } \\
\text { model }\end{array}$ & $\begin{array}{c}\text { Rel.Dev. } \\
\text { in \% }\end{array}$ \\
\hline R1 & 0.1551 & 0.1551 & 0 \\
\hline C1 & 8.758 & 8.758 & 0 \\
\hline R2 & 0.5366 & 0.5368 & -0.04 \\
\hline C2 & 2.356 & 2.352 & 0.26 \\
\hline R3 & 1.374 & 1.374 & 0 \\
\hline C3 & 0.8604 & 0.8604 & 0 \\
\hline R4 & 6.298 & 6.293 & 0.08 \\
\hline C4 & 8.758 & 8.758 & 0 \\
\hline R5 & 0.8214 & 0.5174 & 58.8 \\
\hline C5 & 0.01086 & 0.0111 & -2.2 \\
\hline
\end{tabular}

Table 1 Circuit components with ideal operational amplifier and with operational amplifier model $\omega_{t}=312.5$

\begin{tabular}{|c|c|c|}
\hline Poles & Real part & Imaginary part \\
\hline & -1.000000 & 0.000000 \\
\hline & $-8.090170 \mathrm{D}-01$ & $5.877844 \mathrm{D}-01$ \\
\hline & $-3.090170 \mathrm{D}-01$ & $9.510595 \mathrm{D}-01$ \\
\hline & $-4.882626 \mathrm{D}+02$ & 0.000000 \\
\hline
\end{tabular}

Table 2 Poles of the circuit response with operational amplifier model $\omega_{t}=312.5$

The operational amplifier model is shown in figure 2 with the unity gain frequency

$$
\omega_{t}=\frac{1}{C}
$$

As the most popular application of Butterworth lowpass filter is as anti aliasing filters for audio signals we have chosen in the normalized case to set $\omega_{t}=5 \mathrm{MHz} / 16 \mathrm{kHz}=312.5$ which gives a model capacitor with the value of $C=1 / \omega_{t}=3.2 \mathrm{mF}$. This corresponds to apply the low cost operational amplifier LF156.

We shall now show some results obtained by reducing the scalar error function $\varepsilon(\mathbf{x})$ from equation (3) to zero. The 


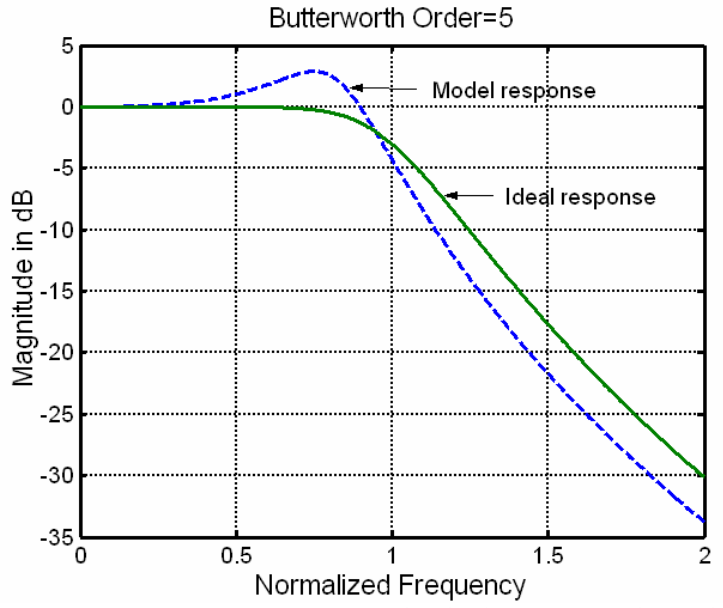

Figure 3 Circuit responses with ideal operational amplifier and with operational amplifier model

optimization process has been started with an initial guess where all the passive component values are set to 1.0.

In Table 1 we have shown the result with the constraint of minimizing the spread of component values both by applying an ideal operational amplifier and by applying an operational amplifier described by the integrator model with a unity gain frequency of $\omega_{t}=312.5$ The corresponding poles are shown in table 2. We observe a non dominant pole situated far outside the pass band of the Butterworth filter. This non dominant pole is caused by the unity gain frequency of the operational amplifier. The unity gain frequency also makes a shift of the dominant poles but this shift is compensated by a change of

the resistors $R_{2}, R_{4}, R_{5}$ and the capacitors $C_{2}, C_{5}$. This can be seen from the $4^{\text {th }}$ column of table 1 where we have shown the relative deviation of the component values compared with the ideal design. In fact by using the nominal component values from the ideal filter the influence of the unity gain frequency may be almost outperformed just by trimming the value of resistor $R_{5}$.

\section{SENSITIVITY ANALYSIS}

Manufactured filters cannot guarantee to correspond exactly to the designed filter performances. The effect of component tolerances should be analyzed. The simplest way of predicting the effect of component tolerances is to use the concept of network sensitivity assuming that the component changes are small. The magnitude variability with respect to the passive components and the unity gain frequency $\omega_{\mathrm{t}}$ is given by

$$
\frac{\Delta|H(\mathbf{x}, s)|}{|H(\mathbf{x}, s)|}=\sum_{i=1}^{5} S_{C_{i}}^{|H|} \cdot \frac{\Delta C_{i}}{C_{i}}+\sum_{i=1}^{5} S_{R_{i}}^{|H|} \cdot \frac{\Delta R_{i}}{R_{i}}+S_{\omega_{t}}^{|H|} \cdot \frac{\Delta \omega_{t}}{\omega_{t}}
$$

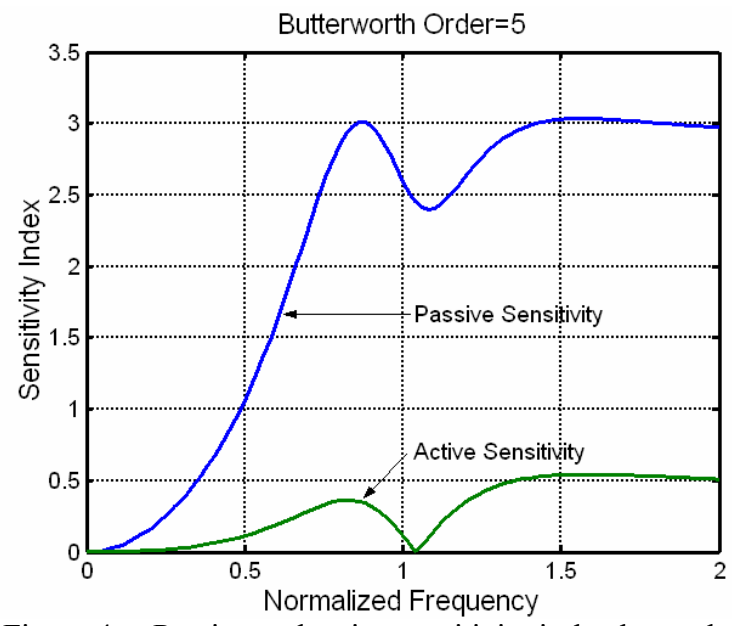

Figure 4 Passive and active sensitivity index by applying the operational amplifier model $\omega_{t}=312.5$

$S_{C_{i}}^{|H|} i=1,2,3,4,5$ are the relative sensitivities of the magnitude function with respect to the five capacitors and $S_{R_{i}}^{|H|}$ $i=1,2,3,4,5$ are the same sensitivities with respect to the resistors. $S_{\omega_{t}}^{|H|}$ is the relative sensitivity with respect to the unity gain frequency. Now we approximate the upper limit of the variability by the following sum

$$
\left.\frac{\Delta|H|}{|H|}\right|_{\text {Schoeffler }}=\sqrt{\sum_{i=1}^{5}\left|S_{C_{i}}^{|H|} \cdot \frac{\Delta C_{i}}{C_{i}}\right|^{2}+\sum_{i=1}^{5}\left|S_{R_{i}}^{|H|} \cdot \frac{\Delta R_{i}}{R_{i}}\right|^{2}+\left|S_{\omega_{t}}^{|H|} \cdot \frac{\Delta \omega_{t}}{\omega_{t}}\right|^{2}}
$$

We have chosen to subdivide the Schoeffler criterion in two parts a passive part with contribution only from the passive components

$$
\left.\frac{\Delta|H|}{|H|}\right|_{\text {passive }}=\sqrt{\sum_{i=1}^{5}\left|S_{C_{i}}^{|H|} \cdot \frac{\Delta C_{i}}{C_{i}}\right|^{2}+\sum_{i=1}^{5}\left|S_{R_{i}}^{|H|} \cdot \frac{\Delta R_{i}}{R_{i}}\right|^{2}}
$$

and an active part with contribution only from the unity gain frequency of the operational amplifier 


$$
\left.\frac{\Delta|H|}{|H|}\right|_{\text {active }}=\left|S_{\omega_{t}}^{|H|} \cdot \frac{\Delta \omega_{t}}{\omega_{t}}\right|
$$

We have chosen this way of subdividing the Schoeffler criterion as we may expect a much larger tolerance on the unity gain frequency of the operational amplifier compared with the tolerance of the passive components. But we can decrease the relative sensitivity $S_{\omega_{t}}^{|H|}$ by choosing an operational amplifier with a higher unity gain frequency.

A sensitivity analysis was performed assuming the relative changes of the resistors, capacitors and the unity gain frequency to be uncorrelated random variables with a zero mean Gaussian distribution and 1\% standard deviation. The Schoeffler sensitivity index which is an estimate of the upper limit of the gain variability in $\%$ is shown on figure 4 .

To obtain an analytical expression of the Schoeffler sensitivity index of the $5^{\text {th }}$ order Butterworth filter in terms of the component parameters is a complicated task. In the limit when the Laplace variable $s \rightarrow \infty$ we may in the all-pole filter case find the following transfer function (The Butterworth filter is a special case of the all-pole filter)

$$
\left.H(s)\right|_{s \rightarrow \infty} \approx \frac{1}{s C_{1} R_{1} s C_{2} R_{2} s C_{3} R_{3} s C_{4} R_{4} s C_{5} R_{5}}
$$

This corresponds to a gain slope of $100 \mathrm{~dB} /$ decade. The passive sensitivity index of the gain function approaches

$$
\left.\frac{\Delta|H| \mid}{|H|}\right|_{\text {passive }} \approx \sqrt{\sum_{i=1}^{5}\left|-\frac{\Delta C_{i}}{C_{i}}\right|^{2}+\sum_{i=1}^{5}\left|-\frac{\Delta R_{i}}{R_{i}}\right|^{2}}
$$

This means that the passive Schoeffler sensitivity index approaches $\sqrt{10} \%=3.16 \%$ with $1 \%$ standard deviation on each of the passive component. This is also the limiting value of the sensitivity index shown on fig. 4 when $\omega \rightarrow \infty$. The overall sensitivity index is below 3.16 for $0 \leq \omega \leq \infty$. We will not claim that this is a minimum value but we regard this as a quite satisfactory result.

This sensitivity index is lower than the sensitivity index reported in [1]. The only difference is due to the fact that we have chosen a unity gain amplifier and have minimized the component spread. But as can be seen from table 1 the component spread is quite high with a value of 790 . This value is too large for integrated circuit design but acceptable for the design of a discrete circuit.
By applying the same method as described above on a unity gain $4^{\text {th }}$ and $3^{\text {rd }}$ order Butterworth filter we have found a component spread of 44 and 7.3 with similar acceptable passive and active sensitivities. In another paper we will describe how the overall component spread may be reduced by introducing less feedback to the operational amplifier. The drawback in this case is an increased passive sensitivity.

\section{CONCLUSION}

A numerical design method has been presented for the design of an all-pole Butterworth filter of order 5 applying just one operational amplifier. The operational amplifier is described by the integrator model. We have shown that it is possible to reduce the influence of the operational amplifier just by trimming one of the resistors in the structure. By reducing the component spread we have found that the Schoeffler sensitivity index takes the maximum value at the slope of the Butterworth characteristic. This is a highly desirable result for a practical realization of the filter. The disadvantage is the component spread which makes this filter impractical for integrated circuit application but well suited for a discrete realization. By applying the same design method in the $4^{\text {th }}$ and $3^{\text {rd }}$ order all-pole Butterworth case the component spread is more acceptable for integrated circuit design.

\section{REFERENCES}

D. Jurisic. G.Moschytz and N.Mijat, "Single Amplifier, Active RC, Butterworth Filters Using Impedance Tapering" Proceedings of the IASTED International Conference Signal Processing, Pattern Recognition, and Application,2002

[2] Rolf Schaumann and Mac E. Van Valkenburg: "Design of Analog Filters" Oxford University Press 2001.

[3] H.Gaunholt and B.Guldbrandsen, "The Design of High-Q SallenKey Biquads with Unity Gain Buffer Amplifiers" International Journal of Circuit Theory and Applications, Vol25, 307-316, 1997

[4] B.A.Kramer: "Unity Gain Third Order Lowpass Filters" Electronic Letters $25^{\text {th }}$ May 1995. Vol.31, No11

[5] H.Gaunholt, "On the Compensation of Active Filters for the Application of Real Operational Amplifiers" Proceeding of the Fourth International Symposium on Network Theory, Ljubljana, Yugoslavia, Sept. 4-7, 1979

[6] L.P.Huelsmann, "Equal-valued-capacitor active RC-network realization of a third order low-pass Butterworth characteristic" Electronic Letters Vol.7, pp271-272,1971

[7] R.Fletcher, "A Modified Marquardt Subroutine for Nonlinear Least Squares" UKAEA Report, 1971

[8] Levenberg, "A method for the solution of certain non-linear problems in least squares" Quart. Appl. Math., 1944, 2, pp. 164-168.

[9] D.W.Marquadt, "An algorithm for least squares estimation of nonlinear parameters", SIAM Journal, Vol. 11, p.431. 